\title{
Lichen sclerosus et atrophicus and sexual abuse
}

Shirley A Warrington, Camille de San Lazaro

\begin{abstract}
Aims-The aetiology of lichen sclerosus et atrophicus (LSA) is unknown. A series of 42 cases of this uncommon condition is reported. The aim of this study was to identify associations of LSA and document the association with sexual abuse. Methods-Information about the patients was obtained by retrospective case note review and some patients were contacted by telephone for further information.

Results-In 12 cases there was evidence of sexual abuse. The abused group were slightly older than the non-abused group but were similar in all other respects. All three patients who presented over the age of 12 years had evidence of sexual abuse. Genital trauma was recalled by the patient or found at examination in 17 cases. Evidence of autoimmunity was present in five cases. Positive microbiological isolates were obtained in 18 cases. In only 11 cases were there no associated factors. The symptoms of LSA started between the ages of 3 and 7 years in most patients. The usual symptoms were related to genital skin involvement, and symptoms related to bladder and bowel function were common (50\%).

Conclusion-In this large series of paediatric LSA, associations with trauma, autoimmunity, and infection were noted. There was a high rate of coexisting sexual abuse with LSA, possibly due to genital trauma.
\end{abstract}

(Arch Dis Child 1996;75:512-516)

Keywords: lichen sclerosus et atrophicus, sexual abuse.

Lichen sclerosus et atrophicus (LSA) is a chronic condition of the epithelium and dermis which is characterised by ivory or white shiny macules and papules that form homogeneous hypopigmented areas. The affected skin shows a tendency to fine wrinkling. A characteristic 'figure of eight' pattern is seen when both the vulva and the perianal areas are involved. Involved skin is sharply demarcated from surrounding normal skin. Hymenal tissue is uninvolved and should appear smooth and symmetrical.

The condition may affect all areas of the body, at all ages, and in both sexes. It is most frequently found in the fifth and sixth decades, but also affects prepubertal girls and premenopausal women. An increased risk of malignancy (squamous cell carcinoma) has been found in adult women, ${ }^{12}$ and long term surveillance is suggested. $^{3}$ Boys as well as men may be affected. In boys LSA may cause phimosis. Adult men are also affected on the penis or elsewhere, and in older men it is the commonest cause of balanitis xerotica obliterans (a possibly premalignant condition). In young girls LSA may be asymptomatic or the lesions may itch, become infected, or cause pain and bleeding. Common presenting symptoms are vulvovaginitis, pruritis, bleeding, blistering, pain on defecation and urinary symptoms. ${ }^{4-6}$ LSA is managed with good hygiene, bland emollients, and mild corticosteroids. The aim of treatment is generally to control symptoms, and the condition often improves at puberty. The aetiology of LSA is unknown. In some patients there is evidence to implicate genetic, ${ }^{4-9}$ hormonal, ${ }^{10}$ and autoimmune related factors. ${ }^{211-14}$ It is well recognised that lichen sclerosus may occur at sites of trauma. ${ }^{15}$

LSA may be mistaken for acute trauma because the involved skin is friable and prone to bleeding from trivial trauma. Unexplained or unexpected bleeding or purpura may suggest that a significant injury has occurred. This has resulted in patients with LSA being the subject of child sexual abuse investigations. ${ }^{816-19}$ The differential diagnosis of abuse and LSA is an important issue. We therefore evaluated the case records of all our patients with LSA for evidence of abuse and other associated factors.

\section{Patients and methods}

Forty two patients with the established diagnosis of LSA were identified by retrospective review of 1617 readily accessible case notes. These notes are held securely by one of us (CSL) for a variety of reasons. This population represented a substantial proportion of the patients referred to the paediatric forensic team over a seven year period (1989-95) and all patients had undergone a genital examination. These patients were referred to one of the authors (CSL) for assessment of suspected child sexual abuse, advice on management of LSA, or investigation of genital symptoms.

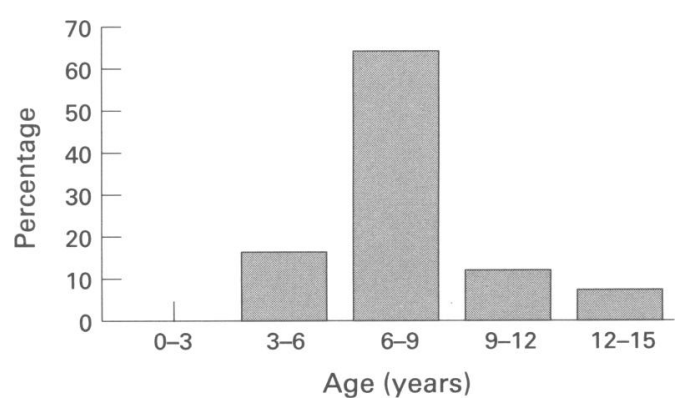

Figure 1 Age distribution at diagnosis. 
The assessment for children referred to the clinic usually involves a long consultation with a consultant paediatrician (up to 90 minutes) and includes a clinical history, general physical examination, and a genital examination. ${ }^{20}$ Some patients were also seen at a special paediatric infectious diseases clinic where appropriate infection screening was carried out as previously described. ${ }^{21}$

The case notes of the 42 cases of LSA were carefully reviewed for demographic details, source of referral, presenting symptoms, further details of the history, and family history. The main reasons for referral were divided into three categories: management of LSA, advice on genital symptoms, and suspected child sexual abuse. Evidence was sought for associated factors, particularly infection, autoimmune disease, and trauma, including sexual trauma from abuse. Details of examination findings and the person making the initial diagnosis were documented. In those cases in which a child sexual abuse inquiry had occurred, case conference minutes were studied and outcome data sought. Additional information about past medical history and family history was obtained from some parents when this was practicable.

\section{Results}

Forty two girls with LSA were identified by case note review. All but two were white. Age of symptom onset ranged from a few months to 13 years. Age at diagnosis ranged from 3.9 to 14.0 years with a mean age of 7.5 years (fig 1 ).

REFERRAL SOURCES

General practitioners referred 16 cases, paediatricians 17 cases, and child protection agencies five cases. The remaining four were referred by other specialists, including one by a cardiac surgeon, when lesions suggesting sexual trauma were noted at urethral catheterisation before surgery.

Diagnosis before referral had been made by three general practitioners and by 10 of the referring paediatricians. In one case a retrospective diagnosis was made from photographs taken in a previous child abuse inquiry. This child also showed gross hymenal damage (fig 2).

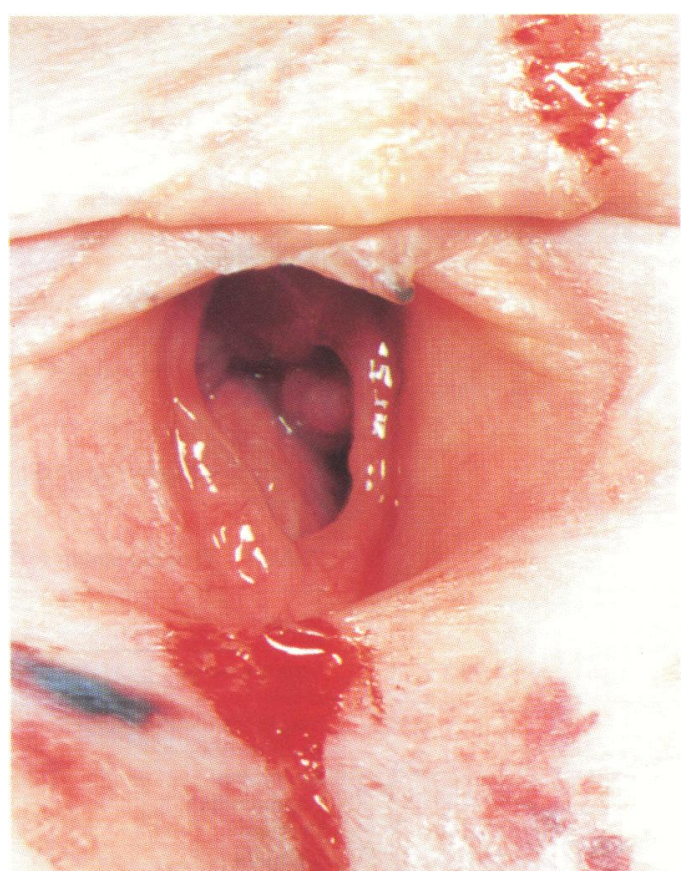

Figure 2 Case 2: 7 year old girl alleging penetration by two family members. A guilty plea was obtained from one and conviction of the other. Healed posterior transection and scarred attenuated left free edge of hymen. Pallor, friability, and bleeding from lesions of $L S A$.

The reasons for referral differed, general practitioners referring for symptom assessment, and paediatricians and child protection agencies referring because of suspected child sexual abuse.

\section{ASSOCIATED FACTORS (FIG 3)}

Injury

A history of accidental trauma or findings of abusive genital trauma were associated with the development of LSA in 17 children. The history of accidental trauma, obtained in six cases, took the form of a variety of memorable falls recalled by the child or parent, preceding the development of LSA. One of these children was also sexually abused. Details of the 12 children in whom evidence of sexual abuse was found are shown in table 1 . Of these, six made allegations resulting in convictions in two. The other six showed damaged hymens and other physical and behavioural signs. Concern about three other children, one with a short tear to

Table 1 Details of cases with evidence of sexual abuse

\begin{tabular}{|c|c|c|c|c|}
\hline $\begin{array}{l}\text { Case } \\
\text { No }\end{array}$ & $\begin{array}{l}\text { Age } \\
\text { (years) }\end{array}$ & Allegation & Findings & Comments \\
\hline 1 & 6 & Yes & Thickened scar across hymen. Disrupted hymen & Conviction obtained \\
\hline 2 & 7 & Yes & $\begin{array}{l}\text { Healed posterior transection and scarred attenuated left } \\
\text { free edge of hymen. Shown in fig } 2\end{array}$ & $\begin{array}{l}\text { Conviction obtained. Father convicted of indecent assault on } \\
\text { child. Grandfather-offences of indecent exposure }\end{array}$ \\
\hline 3 & 13 & Yes & Bruising inner thighs - no adequate explanation & $\begin{array}{l}\text { Violence in home. Brother physically abused and showing } \\
\text { sexualised behaviour }\end{array}$ \\
\hline 4 & 7 & Yes & Intact hymen. Anal laxity with distortion & $\begin{array}{l}\text { Father arrested for allegations of sexual abuse against uncle, aunt, } \\
\text { and another boy }\end{array}$ \\
\hline 5 & 4 & No & $\begin{array}{l}\text { Attenuated hymen with rolled edge. Disruption and } \\
\text { nodular scarring }\end{array}$ & $\begin{array}{l}\text { Proven in court. Withdrawn behaviour. Father had record of } \\
\text { indecent exposure }\end{array}$ \\
\hline 6 & 6 & No & Hymen tethered to labia minora? Post-traumatic adhesions & Highly sexualised behaviour. Nightmares and sleepwalking \\
\hline 7 & 7 & No & $\begin{array}{l}\text { Thick adhesion from inferior free edge of minora to the } \\
\text { hymen }\end{array}$ & $\begin{array}{l}\text { Spent long periods unsupervised with known abuser } \\
\text { (stepgrandfather). Mother also sexually abused by him. Police } \\
\text { investigation }\end{array}$ \\
\hline 8 & 7 & Yes & Nodular hymen with cleft & Exposed to inappropriate sexual activity \\
\hline 9 & 13 & No & Transected hymen with scarring & \\
\hline 10 & 7 & No & Disrupted hymen & \\
\hline 11 & 9 & No & Scar at midline of hymen with deep cleft and attenuation & Possible sexual abuse in stepfather's previous family \\
\hline 12 & 14 & Yes & Widely disrupted hymen & \\
\hline
\end{tabular}




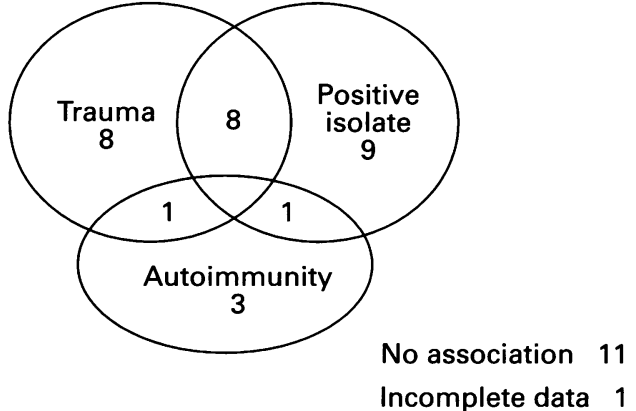

Figure 3 Inter-relationship of associated factors in 42 patients.

Table 2 Positive isolates

\begin{tabular}{ll}
\hline Isolate & No of children \\
\hline Streptococcus & 9 \\
E coli & 4 \\
Staphylococcus & 3 \\
Bacteroides & 2 \\
Anaerobes & 2 \\
Gardnerella & 1 \\
Proteus & 1 \\
Candida & 1 \\
Herpes simplex & 1 \\
Herpes zoster & 1 \\
Echovirus & 1 \\
\hline
\end{tabular}

the hymen, one exposed to a known offender, and one with genital herpes simplex, has not so far developed into a finding of sexual abuse.

\section{Autoimmune disease}

Thirty one case notes yielded information about autoimmune disease. One child had extensive morphoea and two had arthritis. Three other children were serum positive for autoantibodies (one $\mathrm{Rh}$ factor, one antimitochondrial, and one thyroid antibodies). Of the 33 cases where a full family history had been taken, nine were positive for a first or second degree relative $(27 \%$ of those evaluated).

\section{Infection screening}

Results were available in 32 cases of which 21 isolates were seen in 18 children. Warts were not seen in any child. Six children had more than one infectious agent identified. Streptococcus, staphylococcus, Escherichia coli, and bacteroides were isolated from both groups of children. Candida and gardnerella were only isolated in the group of children where abuse had occurred (table 2 ).

SYMPTOM HISTORY

The frequency of presenting symptoms differed between the 12 children who were known to have been abused and the 30 children where abuse was not found.

Table 3 Frequency of presenting symptoms in non-abused and abused cases

\begin{tabular}{llll}
\hline & \multicolumn{2}{l}{ Incidence (\%) } \\
\cline { 2 - 4 } Presenting symptoms & Non-abused $(n=30)$ & Abused $(n=12)$ & All $(n=42)$ \\
\hline Vulvovaginitis & $22(73)$ & $6(50)$ & $28(67)$ \\
Bleeding & $13(43)$ & $3(25)$ & $16(38)$ \\
Pruritis & $9(30)$ & $1(8)$ & $10(24)$ \\
Urinary & $8(27)$ & $4(33)$ & $12(29)$ \\
Bowel symptoms & $7(23)$ & $4(33)$ & $11(26)$ \\
Purpura/blisters & $6(20)$ & $1(8)$ & $7(17)$ \\
Other & $1(3)$ & $1(8)$ & $2(5)$ \\
None & $2(7)$ & $2(17)$ & $4(10)$ \\
\hline
\end{tabular}

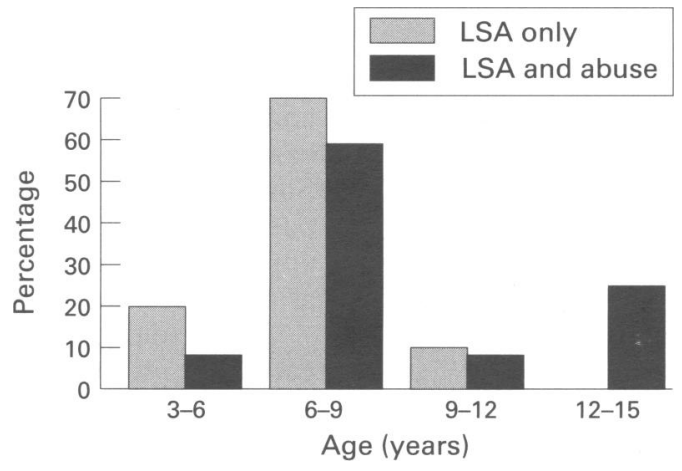

Figure 4 Age at diagnosis of LSA with and without abuse.

Abused children with LSA presented less often with genitourinary symptoms and also presented later (abused children with vulvovaginitis $50 \%$, others $73 \%$; abused children with bleeding $25 \%$, others $43 \%$.) Some $33 \%$ of the abused group presented first with bowel symptoms (pain on defecation and constipation) compared with $23 \%$ of the others. Only $8 \%$ of abused girls were diagnosed with LSA before 6 years of age, compared with $23 \%$ in the non-abused group. About $25 \%$ of the abused girls presented with LSA for the first time after the age of 12 years. All the non-abused girls presented before this age (fig 4).

Table 3 shows the frequency of presenting symptoms. The majority of patients presented with symptoms related to genital skin involvement, soreness, bleeding, discharge, and pruritis. Symptoms relating to bladder and bowel function were seen in $50 \%$ of cases. These symptoms, which included dysuria, dribbling, and stool withholding, appeared to be related to the presence of open tender areas of fissuring and resolved rapidly with treatment. Some patients were asymptomatic, and LSA was discovered incidentally in four cases.

The reasons for referral were compared with outcome diagnosis. Of 12 children referred for symptoms alone, one was found to have LSA coexisting with sexual abuse. Of 12 girls referred for suspected sexual abuse, three were found to have LSA alone and nine were confirmed to have been abused. Eighteen children were referred with the established diagnosis of LSA, and in two of these coexisting sexual abuse was found (fig 5).

\section{Discussion}

The incidence of LSA remains unknown, even in adults. Wallace suggested that the frequency varies between one in 300 to less than one in 1000 of all new patients in a general hospital, depending on the liaison between the skin and gynaecological departments. ${ }^{1}$ The disease is much commoner in adults than in children: in a series of 290 cases only $7 \%$ occurred below the age of $16 .^{1}$ No surveys have been carried out to establish how commonly the condition occurs. In Newcastle, the Lindisfarne Centre is a tertiary referral centre with a specialist population. Seventeen of our cases came from Newcastle with a population of 280000 . These did not represent all the LSA in Newcastle 
42 patients with LSA

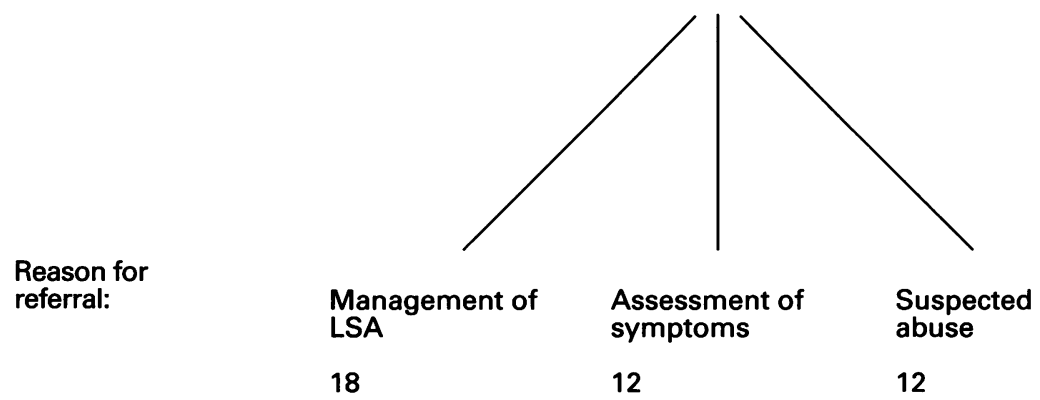

Final

diagnosis:

$\begin{array}{llll}\text { LSA only } & 16 & 11 & 3 \\ \text { LSA and abuse } & 2 & 1 & 9 \\ \text { Abuse only } & 0 & 0 & \text { N/A }\end{array}$

Figure 5 Reason for referral compared with final diagnosis.

children. Incidence rates are fraught with difficulty, and they may be an underestimate of the true rate as mild cases may be missed and patients may present to other specialists. Paediatricians may see the occasional case of lichen sclerosus but some may never see a single case. It may present to other specialists such as dermatologists, gynaecologists, or professionals working in the field of child abuse. We have attempted to compare numbers with those found in Leicester ${ }^{6}$ but our referrals are made to a paediatrician with an interest in this condition. They come from a specialised population of children, many of whom have been referred with vulvovaginitis or other genitourinary symptoms, or for assessment of suspected sexual abuse. In Newcastle the referral patterns have changed over the years. An increased awareness of potential sexual abuse may have resulted in cases being referred directly to the specialist paediatrician (CSL) rather than to urologists, surgeons, or dermatologists. Two of the children referred locally were managed in conjunction with the dermatologists, as were many of the cases which were returned to the referring centre. This pattern of joint care enables the expertise of both specialties to be combined and is particularly appropriate in difficult cases.

The peak age for diagnosis of LSA in our series was found to be 6 to 8 years with $64 \%$ of patients diagnosed then. This is the peak age for atrophic vulvitis, when oestrogen concentrations are at their nadir. No cases of LSA were diagnosed below the age of 3 years. The influence of maternal oestrogens lasts until the age of 2 years and this may be a protective factor explaining the rarity of LSA below this age.

All the 42 patients in this series were female. The condition is known to be much commoner in girls than boys. Histological evidence of balanitis xerotica obliterans (as the condition is often termed in the male) can frequently be found after circumcision for scarring phimosis. ${ }^{22}$ Thus boys with LSA are more likely to be referred to urologists or surgeons than to paediatricians. Berth-Jones et al reported that two thirds of their patients presented with soreness, ${ }^{6}$ a figure similar to our numbers presenting with vulvovaginitis (a syndrome including soreness and discharge). The Leicester group found that urinary symptoms occurred in $66 \%$ of their patients and bowel symptoms in $50 \%{ }^{6}$ as compared with half that incidence of symptoms reported by our patients. Chernosky et al found that pruritis was described in $49 \%$ of cases, ${ }^{15}$ whereas we found that only half that number of patients reported this symptom. The differing presentation of genital symptoms and bowel symptoms in children with LSA and abuse raises the possibility that symptoms, particularly relating to the genital area, may be unreported by children or families involved in sexual abuse, whereas bowel symptoms may be perceived as less threatening and also tend more to mandate clinical attention.

Associated factors were commonly found with LSA in our patients. It has been suggested that genetic factors are involved in the aetiology of LSA. ${ }^{4-9}$ We found two siblings with the condition. Details of the family history of autoimmune disease showed that the affected family members were generally the patient's grandparents reflecting the younger age of the patient's parents compared with the usual age of onset of these diseases. This is similar to the incidence of autoimmune disease in relatives previously reported, ${ }^{23}$ and adult studies ${ }^{211} 12$ have found a high rate of autoantibodies in patients with LSA. One girl had patchy morphoea. The frequency of occurrence of morphoea in combination with LSA has been described as statistically significant. ${ }^{1}$

Positive microbiological isolates have not previously been reported in LSA. In this series positive swabs were obtained in 18 cases (56\% of those tested) and negative ones in 14 cases. In a comparable normal population the rate of carriage of isolates is unknown. However, these figures are considerably higher than those found in a previous study carried out by this department where only $4 \%$ of children had positive isolates. ${ }^{21}$ The high rate of positive isolates is likely to relate to secondary colonisation of compromised damaged skin in LSA. We have no evidence that these positive isolates are causative, but all positive isolates were treated, as potentially the children required the application of local topical steroids to treat the LSA. The presence of candida and gardnerella has been associated with sexual abuse, ${ }^{21}{ }^{24}$ and our findings support this suggestion. No warts were found in any of our cases, in spite of the finding of Kiene $e t$ al of four of 18 cases of LSA being infected with human papillomavirus type $16 .^{25}$ More specific testing for wart virus in children with LSA may provide further evidence of this possible association.

Early literature notes the occurrence of the Koebner phenomenon in patients with LSA. ${ }^{15}$ Chronic frictional injury from waist bands and bra straps is well known to precipitate the appearance of lichen sclerosus lesions. ${ }^{26} \mathrm{Har}-$ rington reports extragenital lesions occurring at sites of acute trauma-for example, oven burns. ${ }^{26} \mathrm{Six}$ of our patients could recall a definite accidental injury to the genital area. Some 
authors have suggested that LSA is secondary to sexual abuse $\mathrm{e}^{26-28}$ while others have disputed this. ${ }^{29}$ In 12 of our patients there was evidence of such abuse. In one of the children who had been abused there was also a history of accidental injury preceding by months the development of the disease. The changes seen in LSA in the abused group may be triggered by intermittent frictional trauma to the genital area. However, we believe that the recurrent presence of foreign substances, like semen, saliva, or lubricants, should be also considered a possible trigger.

There are numerous reports of LSA being mistaken for childhood sexual abuse. ${ }^{816-19}$ In our study this occurred in three cases. The issue raised by these cases remains, as always, that of the role of physical findings in formulating an opinion about abuse. The sexually abused victim commonly demonstrates no physical signs, and such findings as there are rarely reflect the degree of trauma that children report. Most detected physical abnormalities at the genital area are neither amenable to dating nor to understanding of chronicity. A single event of digital penetration in a small child could be reflected in gross abnormality years later; frequent, painful friction between the labia may result in minimal, if any, physical abnormality. These limitations underpin difficulties practitioners experience when an incidental finding of physical abnormality in an apparently well adjusted symptom free child is made. In practice it is rarely easy to design a meaningful child protection process in these situations. Our clinical experience of over 2500 sexually abused children suggests that it is unusual to see superficial skin tears, bleeding, and bruising of external structures in acute sexual assault with no disclosure or signs of hymenal trauma. This is an unusual constellation of signs for sexual abuse and would mitigate against this diagnosis. The combination of these signs is peculiar to LSA. Evidence of abuse was found in three of the patients referred for symptom assessment or management of LSA. One of these patients was already known to have been abused, giving two 'new' cases of abuse discovered within the whole group of 42 children.

The unexpectedly high numbers of children with LSA existing with sexual abuse would suggest that some attention should be paid to the possibility of coexistence. Our practice in both children with LSA and abused children is to screen for infections, for obvious reasons in the latter and for reasons of prudence in the former because of the potential need for topical steroids.

Children with findings of hymenal trauma are referred for formal multiagency investigation. There remains the question of what should be done when children present with pure findings of LSA. We do not believe that this study's findings mandate a child abuse inquiry in all children with LSA. Our practice is to review all children frequently to monitor symptoms and treatment efficacy. Over this period sensitive and gentle phased exploration of other behavioural symptoms or family factors is usually possible. Where other significant concerns come to light, the matter may be drawn into a wider multidisciplinary forum. When no such concerns arise, LSA remains a 'stand alone' diagnosis.

In conclusion, LSA is commoner in our practice than previously reported. It frequently has associations with trauma and/or autoimmune disease. There is a definite association with sexual abuse with which the condition may be confused. In this series of children with LSA, serious concerns about sexual abuse exist in 15 of 42 children ( $36 \%$ ), and a finding of sexual abuse was made in 12 of 42 cases seen $(29 \%)$. Previously there have only been isolated case reports of this association. ${ }^{26-28}$ This is the first detailed description of the characteristics of these cases.

1 Wallace HJ. Lichen sclerosus et atrophicus. Transactions of $S t$ fohn's Hospital Dermatology Society 1971; 57:9-30.

2 Meyrick Thomas RH, Ridley CM, McGibbon DH, Black MM. Lichen sclerosus et atrophicus and autoimmunity-a study of 350 women. Br $\mathfrak{f}$ Dermatol 1988;118:41-6.

3 Ridley CM. Lichen sclerosus et atrophicus. BMF 1987;295: 1295-6.

4 Tremaine RDL, Miller RAW. Lichen sclerosus et atrophicus. Int $\mathcal{F}$ Dermatol 1989;28:10-6.

5 Clark JA, Muller SA. Lichen sclerosus et atrophicus in children: a report of 24 cases. Arch Dermatol 1967;95:47682.

6 Berth-Jones J, Graham Brown RAC, Burns DA. Lichen sclerosus et atrophicus-a review of 15 cases in young girls. Clin Exp Dermatol 1991;16:14-7.

7 Meyrick Thomas RH, Kennedy CTS. The development of lichen sclerosus et atrophicus in monozygotic twin girls. $B$ f Dermatol 1986;114:377-9.

8 Davidson DC, Clarke MDB, Kean HB. Lichen sclerosus et atrophicus in children misdiagnosed as sexual abuse [letter]. BMF 1987;295:211.

9 Shirer JA, Ray MC. Familial occurrence of lichen sclerosus et atrophicus. Arch Dermatol 1987;123:485-8.

10 Friedrich EG, Kalra PS. Serum levels of sex hormones in vulvar lichen sclerosus, and the effect of topical testostervulvar lichen sclerosus, and the effect

11 Goolamali SK, Barnes EW, Irvine WJ, Shuster S. Organspecific antibodies in patients with lichen sclerosus. $B M^{\mathscr{F}}$ specific antibodic

12 Harrington CI, Dunsmore IR. An investigation into the incidence of autoimmune disorders in patients with lichen sclerosus et atrophicus. Br ₹ Dermatol 1981;104:563-6.

13 Meyrick Thomas RH, Ridley CM, Black MM. The association of lichen sclerosus et atrophicus and autoimmunerelated disease in males. $B r \mathcal{F}$ Dermatol 1983;109:661-4.

14 Lavery HA, Pinkerton JHM, Callender M. The associatio of lichen sclerosus et atrophicus and primary biliary cirrhosis. Br f Dermatol 1985;112:729-30.

15 Chernosky ME, Derbes VJ, Burks JW. Lichen sclerosus et atrophicus in children. Arch Dermatol 1957;75:647-52.

16 Handfield-Jones SE, Hinde FRJ, Kennedy CTC. Lichen sclerosus et atrophicus in children misdiagnosed as sexual abuse. BMF 1987;294:1404-5.

17 Priestley BL, Bleehen SS. Lichen sclerosus et atrophicus in children misdiagnosed as sexual abuse [letter]. BMF 1987; 295:211.

18 Jenny C, Kirby P, Fuquay DM. Genital lichen sclerosus mistaken for child sexual abuse. Pediatrics 1989;83:597-9.

19 Bays J, Carol J Genital and anal conditions confused with child sexual abuse trauma. Am ₹ Dis Child 1990;144:1319.

20 de San Lazaro C. Making paediatric assessment in suspected sexual abuse: a therapeutic experience. Arch Dis Child 1995;73:174-6.

21 Steele AM, de San Lazaro C. Transhymenal cultures for sexually transmissible organisms. Arch Dis Child 1994;71: 423-7.

22 Chalmers RJG, Burton PA, Bennett RF, Goring CC, Smith PJB. Lichen sclerosus et atrophicus. Arch Dermato 1984;120:1025-7.

23 Ridley CM. Genital lichen sclerosus (lichen sclerosus et atrophicus) in childhood and adolescence. $\mathcal{F} R$ Soc Med 1993;86:69-75.

24 Argent AC, Lachman PI, Hanslo D, Bass D. Sexually transmitted diseases in children and evidence of sexual abuse. Child Abuse Negl 1995;19:1303-10.

25 Kiene $\mathrm{P}$, Milde-Langosch $\mathrm{K}$, Lönig T. Human papillomavirus infection in vulvar lesions of lichen sclerosus et atrophicus. Arch Dermatol Res 1991;283:445-8.

26 Harrington CI. Lichen sclerosus [letter]. Arch Dis Child 1990;65:335

27 Priestley BL, Bleehen SS. Lichen sclerosus and sexual abuse [letter]. Arch Dis Child 1990;65:335.

28 de San Lazaro C. Lichen sclerosus [letter]. Arch Dis Child 1990;65:1184.

29 Berth-Jones J, Graham Brown RAC, Burns DA. Lichen sclerosus. Arch Dis Child 1989;64:1204-6. 